\title{
Radio-frequency actuated polymer-based phononic meta-materials for control of ultrasonic waves
}

\author{
Ezekiel L Walker ${ }^{1,2}$, Zhiming Wang ${ }^{1}$ and Arup Neogi ${ }^{1,2}$
}

Radio-frequency (RF) control of an ultrasonic phononic crystal was achieved by encapsulating it in a composite of high k-10\% $\mathrm{KF}$-doped $\mathrm{BaTiO}_{3}$ dielectric nanoparticles with poly $(\mathrm{N}$-isopropylacrylamide) (PNIPAm)-based hydrogel. The combination of the nanoparticles and hydrogel produced a composite with elastic properties susceptible to RF actuation. The novel acoustic meta-material enables the regulation of sound waves by electromagnetic waves, which is not possible in a conventional medium as elasto-mechanical waves, and electromagnetic waves do not directly couple. Compared with light waves, radio waves can penetrate deeper into bulk structures and enable the control of propagation of ultrasonic waves through a macroscale phononic crystal. An RF antenna emitting at 318.6 and $422.5 \mathrm{kHz}$ was used to modulate the device in water and ambient air, respectively. An increased transparency of the ultrasonic wave in the material was observed due to an increase in the bandwidth of the modulated device exceeding $8 \mathrm{kHz}$ with a 30 -fold increase in the signal modulation at select frequencies. The radio waves induced changes in the transmission and demonstrated the control of ultrasound with applied RF. The synthetic acoustic properties in the resultant meta-material device were actively manipulated through the interaction of electromagnetic waves with the material.

NPG Asia Materials (2017) 9, e350; doi:10.1038/am.2016.209; published online 24 February 2017

\section{INTRODUCTION}

Artificially arranged periodic media can produce materials with synthetic properties that control the transient propagation of electrons, light and/or mechanical waves. Periodic arrangements of dielectrics produce photonic crystals that are well-studied, with devices ranging from filters ${ }^{1}$ to superlenses ${ }^{2}$ to cloaks. ${ }^{3}$ Transmission of mechanical waves through phononic crystals is governed by the elastomorphic and material parameters of the crystal. ${ }^{45}$ These crystals require two or more materials with a high contrast in density, attenuation, elasticity or sound velocity and at least one material must have a periodic or semiperiodic arrangement of scatterers. ${ }^{4,6}$ The elastomorphic properties pertaining to the structural features of the phononic crystal include the size, shape, arrangement and orientation of the crystal components. The material parameters include elasto-mechanical properties such as the density, bulk and shear modulus, attenuation, and physical state of a crystal component. As with electronic and photonic crystals, the transmissive evolution of mechanical or elastic waves is revealed in the phononic crystal bandstructure, with many shared synthetic properties across the forms of energy. ${ }^{7-10}$ Transmission stopgaps, ${ }^{9}$ resonators, ${ }^{11}$ and antiparallel group and phase velocity propagation ${ }^{7}$ can all be attained through the appropriate crystal design. Metamaterials are most prominently known for their ability to produce synthetic properties including negative index and 'slow' propagation. ${ }^{7}$ These effects normally occur at the edge of brillouin zones or above the homogenization limit and are revealed in dispersion relation and spectral intraband characteristics such as pseudogaps. ${ }^{12,13}$ Mechanisms that actively modify some combination of the elastomorphic and material parameters of a phononic crystal result in metamaterials and devices where control of transient sound is coupled to the moderating mechanism, as evidenced by the dispersion relation and spectral characteristics.

Active control of a phononic structure by electromagnetic (EM) means is of interest for both the ability to operate without contact and to couple light to the control of propagating elastic waves. Active tuning can be accomplished by altering the synthetic, that is, non-intrinsic features of the phononic crystal such as the density, bulk or shear modulus, or structure of the phononic crystal components. More specifically, modifying the contrast in material parameters of a crystal, or changing the physical arrangement of components, manifests in the transmission spectrum and band structure to show the effects on transient sound. Active sonic structures with applied static electric fields, ${ }^{14-16}$ magnetically, ${ }^{17-19}$ mechanically ${ }^{20,21}$ and recently with infrared radiation ${ }^{22}$ have been reported. Most of these active sonic structures are in physical contact with the actuating source. As phononic crystals range in size from meters ${ }^{23}$ to microns ${ }^{24,25}$ and smaller, ${ }^{26}$ the use of a radio-frequency (RF) EM stimulus in particular allows for bulk and micro-phononic crystal structures to be altered with features maintaining appropriate

${ }^{1}$ Institute of Fundamental and Frontier Sciences, Chengdu, China and ${ }^{2}$ Department of Physics, University of North Texas, Denton, TX, USA

Correspondence: Professor A Neogi, Department of Physics, University of North texas, Denton, TX 76210, USA.

E-mail: arup.neogi@unt.edu

or Professor Z Wang, IFFS, University of Electronic Science and Technology, Chengdu, Sichuan 610051, China.

E-mail: zmhwang@gmail.com

Received 14 July 2016; revised 6 November 2016; accepted 21 November 2016 
RF response. The general EM property of deep penetration into materials makes RF actuation more efficient compared with other mechanisms for long range, contactless tuning or control. In this work, RF stimuli are used to control ultrasound by changing the density and elasto-mechanical properties of a component material of the phononic device with applied RF.

\section{MATERIALS AND METHODS}

\section{Phononic crystals}

Stainless steel welding rods $1.59 \mathrm{~mm}\left(1 / 16^{\prime \prime}\right)$ in diameter and $10.16 \mathrm{~cm}\left(4^{\prime \prime}\right)$ long were arranged in a $1.96-\mathrm{mm}$ square lattice computer numerical control (CNC) milled into a plastic base to form the phononic crystal. The length of rod used for the phononic crystal portion between the base and top is $8.89 \mathrm{~cm}$ (3.5"). The reduced frequency is defined as $\Omega=\omega /\left(c_{l} \cdot k\right)$, where $c_{l}$ is the velocity of sound in the propagating medium. The crystal filling fraction of $51.6 \%$ results in the center of the first stop band occurring at the reduced frequency of $\Omega \approx 0.56$. The result is a first bandgap centered at approximately $410 \mathrm{kHz}$.

An image of the base phononic crystal can be found in the Supplementary Materials. The crystal is $10 \times 20$ periods (propagation direction vs width). The dimensions of the phononic crystal allow (1) confirmation of the ability of RF to tune bulk structures as the total crystal dimension are on the centimeter scale and (2) the effective frequency range of the phononic crystal to be solidly in the $100 \mathrm{kHz}-1 \mathrm{MHz}$ ultrasonic frequency range.

\section{$10 \% \mathrm{KF}$-doped barium titanate $\left(\mathrm{KF}-\mathrm{BaTiO}_{3}\right)$}

A sol-gel technique was used to form nanoparticles of $\mathrm{KF}_{0.1}-\mathrm{BaTiO}_{3}$. The process is described and followed in detail from other works. Titanium tetraisopropoxide $\left(\mathrm{Ti}\left[\mathrm{OCH}\left(\mathrm{CH}_{3}\right)_{2}\right]_{4}\right)$, barium diethoxide $\left(\mathrm{Ba}\left(\mathrm{OC}_{2} \mathrm{H}_{5}\right)_{2}\right)$ and $\mathrm{KF}$ powders were dissolved at a molar ratio of 1.0:0.9:0.1 in sequence in a mixed solution of methanol and 2-methoxyethanol in a dry glove box with an $\mathrm{N}_{2}$ gas flow. Hydrolysis was carried out with distilled water by spraying the solution after being cooled to $0^{\circ} \mathrm{C}$ while being magnetically stirred. The resulting gel was dried at $50^{\circ} \mathrm{C}$ for $24 \mathrm{~h}$, then at $90^{\circ} \mathrm{C}$ for 3 days. It was then pulverized and calcined at $650{ }^{\circ} \mathrm{C}$ to remove organic matter and additionally fired at $800^{\circ} \mathrm{C}$ for $2 \mathrm{~h}$.

\section{Bulk poly(N-isopropylacrylamide) (PNIPAm) hydrogel}

The plain, bulk PNIPAm hydrogel was formed using free-radical polymerization, and the composites utilized a slightly modified free-radical polymerization procedure. For the standard PNIPAm gel, $\mathrm{N}$-isopropylacrylamide monomer (NIPA; TCI Chemicals, Tokyo, Japan), $N, N^{\prime}$-methylene-bisacrylamide crosslinker (BIS; Polysciences Inc., Warrington, PA, USA) and DI water were mixed together in a ratio of $0.10(w t): 0.02(\mathrm{~mol}$ NIPA):0.84 (wt) to create a monomer solution. The solution was then set in an ice bath and pumped with $\mathrm{N}_{2}$ for $30 \mathrm{~min}$ to remove oxygen from the solution. Ammonium persulfate (98+\%; Sigma-Aldrich, St Louis, MO, USA) initiator and $N, N, N^{\prime}, N^{\prime}$-tetramethyl-ethylenediamine ( $99 \%$; Sigma Aldrich, TEMED) accelerator were added for final polymerization.

\section{Poly (vinyl alcohol) (PVA) PNIPAm composites}

For the PVA modified composites, NIPA monomer (TCI Chemicals), N, '-methylene-bisacrylamide crosslinker (BIS; Polysciences Inc.) and DI water were mixed together in a ratio of $0.10(\mathrm{wt}): 0.02(\mathrm{~mol} \mathrm{NIPA}): 0.84(\mathrm{wt})$ to create a monomer solution. PVA(Polysciences Inc.), at $2 \mathrm{wt}$. \% of the total mixture, and KBT nanoparticles, at $1 \mathrm{wt}$. \% of the total mixture, were then added to the monomer solution. The solution was heated to $50^{\circ} \mathrm{C}$ and stirred for $>24 \mathrm{~h}$ to ensure the dissolution of the PVA into the monomer solution and the dispersion of the nanoparticles in the solution. The composite solution was then set in an ice-bath and pumped with $\mathrm{N}_{2}$ for $>1 \mathrm{~h}$ to remove adsorbed oxygen. Ammonium persulfate and TEMED were used as the initiator and accelerator for final polymerization in the PVA PNIPa bulk gels/composites. The final gels were immersed in DI water over 2 days. Since PVA is water soluble, the hydrogel was heated to approximately $50^{\circ} \mathrm{C}$, and the excess liquid was removed and replaced with more DI water. The gel was then allowed to rehydrate and the process was repeated two times for each sample to remove more PVA.

\section{RF-tuned phononic crystal device}

The fabrication of the gel-filled sonic crystals incorporated the KBT and PVA acoustic materials discussed in the prior section. For each case, $40 \mathrm{~g}$ of NIPA (Polysciences, Inc.) $1.09 \mathrm{~g}$ of $-N, N^{\prime}$-methylenebisacrylamide (BIS; Polysciences, Inc.), $160 \mathrm{~g}$ of $5 \%$ DI water diluted PVA(Polysciences, Inc.) and $190 \mathrm{~g}$ of DI $\mathrm{H}_{2} \mathrm{O}$ were mixed together to form a solution with the following ratios: 0.10 (wt.):0.02 (mol. NIPA):0.02 (wt.):0.84 (wt.). The solution was magnetically stirred at $70{ }^{\circ} \mathrm{C}$ for $>24 \mathrm{~h}$ in a sealed container. For KBT800, 10\% KF-doped $\mathrm{BaTiO}_{3}$ nanoparticles calcined at $800{ }^{\circ} \mathrm{C}$ were added to the PVA-water solution before being mixed with the NIPA-BIS monomer solution. The sonic crystals were placed into the monomer solution, and the entire vessel containing both the crystal and the solution was placed in an ice-bath and pumped with $\mathrm{N}_{2}$ while being magnetically stirred at high speed for $>30 \mathrm{~min}$. Ammonium persulfate initiator and $N, N, N^{\prime}, N^{\prime}$-tetramethyl-ethylenediamine accelerator were diluted into water with the ratios 0.1(APS; TEMED):0.9 $\left(\mathrm{H}_{2} \mathrm{O}\right)$ and injected into the SC nanoparticle solution in an $\mathrm{N}_{2}$ atmosphere until the hydrogel polymerized. The excess PVA in the SC-gel devices was diluted out by repeatedly placing the structure in a large bath of $\mathrm{DI}_{2} \mathrm{O}$ that was heated above the lower-critical solution temperature $\left(33^{\circ} \mathrm{C}\right)$ to $40^{\circ} \mathrm{C}$, removed, and then replaced with fresh $\mathrm{DI} \mathrm{H}_{2} \mathrm{O}$. For final preparation, the impinging surfaces of the phononic crystal were cleared of the hydrogel composites.

\section{Ultrasonic spectroscopy}

Ultrasonic spectroscopy was accomplished using a bistatic setup of two $0.5 \mathrm{MHz}$ Panametrics V301 ultrasonic transducers as an emitter and detector on opposing sides of the phononic crystal. A spectral scan was performed using a WaveStation $201010 \mathrm{MHz}$ Waveform Generator sweeping 200-800 kHz.

For ambient water, the spectra was recorded at $5 \mathrm{~min}$ intervals with $\mathrm{RF}$ continuously applied over a 120 min time span on an HP 3585A Spectrum Analyzer (Agilent Technologies, Santa Clara, CA, USA) connected to the detecting transducer. The functional frequency sensitivity of the transducer setup was normalized to water as the standard. For ambient water, the devices were placed in a container capable of holding adequate water to completely submerge the phononic crystals while still fitting between the RF antennae plates without providing a direct electrical conduit through the devices. The phononic crystal is a $20 \times 10$ array of stainless steel rods. In ambient water, transmission was measured with the transducers opposite the 10-period sides of the array. The $450-650 \mathrm{kHz}$ range was of particular focus because it was the only window that contained a full transmission band in the $200-800 \mathrm{kHz}$ measurement window. An example of the transmission spectrum is given in Figure 2.

For ambient air, the spectra was recorded on the RF Spectrum Analyzer channel of a Tektronix MDO3024b (Tektronix, Beaverton, OR, USA). Two $5.9 \mathrm{~cm} \times 3 \mathrm{~cm}$ RF plates emitting at $422 \mathrm{kHz}$ were placed opposite to the 10 period sides of the KBTSC. RF was continuously applied for $50 \mathrm{~min}$ and data were recorded every $2 \mathrm{~min}$. After $50 \mathrm{~min}$, the RF was turned off and data were recorded every $10 \mathrm{~min}$ for an additional $50 \mathrm{~min}$. Transmission was measured using transducers on opposite sides of the 20-period side of the array.

\section{RESULTS}

\section{RF actuated acoustic material}

Polymers based on PNIPAm possess mechanical characteristics that undergo reversible and discontinuous transitions when stimulated. The volumetric phase transition of bulk PNIPAm formed using free radical polymerization results in a reversible ultrasonic sound velocity shift of $>75 \mathrm{~m} \mathrm{~s}^{-1}$ from 1315 to $1400 \mathrm{~m} \mathrm{~s}^{-1}$ and a corresponding increase in stiffness (Figure 1a). ${ }^{27}$ Since the propagation of sound is dependent on the mechanical properties of the material it traverses, these pliable elastic material parameters are ideal for tunable phononic structures. EM stimuli of PNIPAm have been demonstrated using infrared $^{28,29}$ and ultraviolet ${ }^{30,31}$ light but are restricted to relatively small-scale structures. ${ }^{32,33}$ PNIPAm does not, however, innately possess adequate RF sensitivity for RF actuation. Since the primary cause of the change in PNIPAm is heat, the ability of a PNIPAm-based material to couple to radio-frequency stimuli is dependent on the 
a

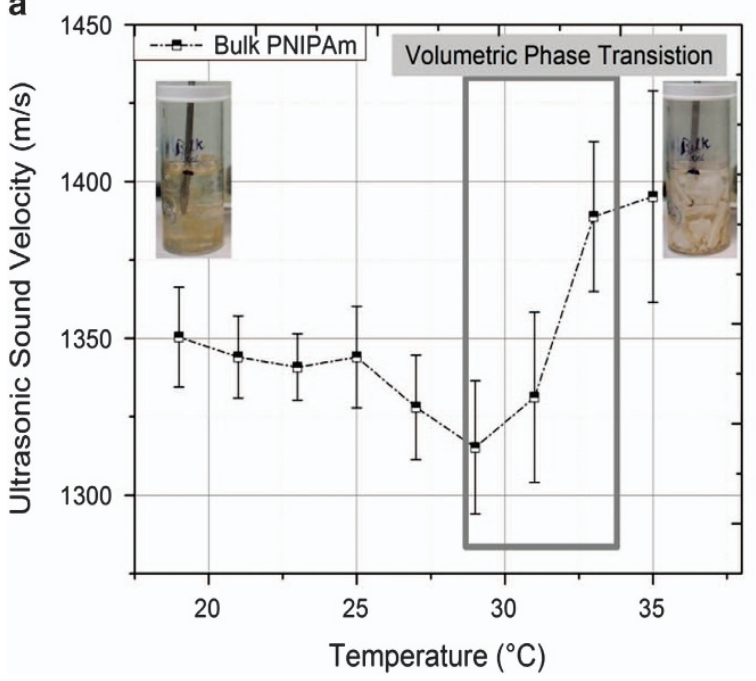

b

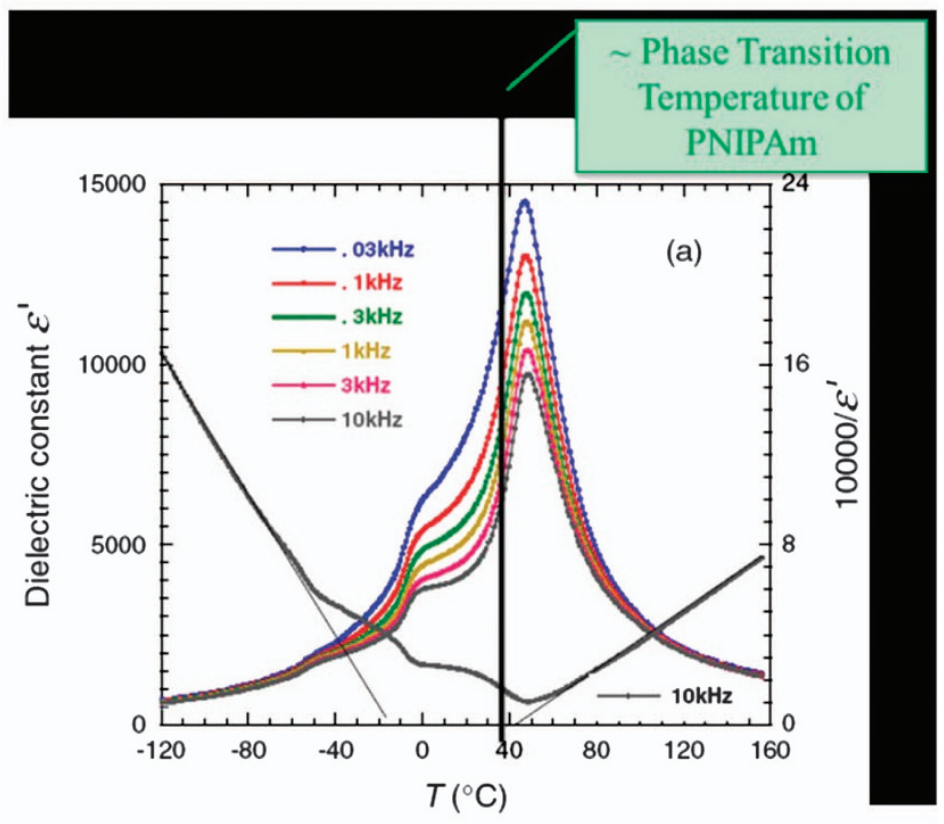

Figure 1 (a) PNIPAm exhibits anomalous dependence of the speed of sound on temperature and dispersion of the ultrasonic sound velocity indicating its nonlinearly varying elastic properties. Dispersion of the ultrasound velocity was inferred from the increased variability in the Fabry-Perot resonances used to derive the sound velocity. ${ }^{24}$ (b) The high dielectric constant $\left(\varepsilon^{\prime}\right)$ of $10 \%$ KF-Doped $\mathrm{BaTiO}_{3}$ spark plasma sintered ceramics in the RF range near the PNIPAm phase transition temperature is utilized for increasing dielectric susceptibility. ${ }^{41}$

dielectric parameters, specific heat and electric field strength inside the material. ${ }^{34,35}$ The relatively weak EM and more specifically the radiofrequency response necessitates increased susceptibility to RF to successfully obtain amplified responsiveness. ${ }^{36-38}$

An applied EM field results in a rearrangement of the charges as they tend to align with the external field. The power density dissipated due to an EM field in a material with low magnetic susceptibility is

$$
p_{\mathrm{m}}=\omega \cdot \epsilon_{0} \cdot \epsilon_{\mathrm{r}}^{\prime} \tan \delta \cdot\left|E_{\mathrm{m}}\right|^{2}
$$

where $\epsilon_{\mathrm{r}}^{\prime}$ and $\tan \delta$ are the dielectric permittivity and loss tangent of the material, and $E_{\mathrm{m}}$ is the average effective electric field inside the material.

It is apparent from equation (1) that ideally, the higher the dielectric constant and loss tangent, the greater the power dissipated inside the material for equal electric fields. Increased susceptibility results from the appropriate additive material that amplifies the dielectric response in the frequency range of interest. The additive material must not destabilize the polymer or the combination of the two will disrupt or eliminate the desired elastic properties. $\mathrm{BaTiO}_{3}$ ceramics doped with varying amounts of $\mathrm{KF}$ to produce KF-doped barium titanate $\left(\mathrm{Ba}_{x} \mathrm{~K}_{1-x} \mathrm{TiO}_{x} \mathrm{~F}_{1-x}\right)$ nanoparticles result in ceramics and powders that exhibit enhanced dielectric and piezoelectric properties in the RF frequency range. ${ }^{39-41}$ The addition of $\mathrm{KF}$ to $\mathrm{BaTiO}_{3}$ causes the compound to locate near the tri-critical point, which enhances the fluctuation of the crystal polarization. Additionally, $10 \% \mathrm{KF}$-doped $\mathrm{BaTiO}_{3}\left(\mathrm{Ba}_{0.9} \mathrm{~K}_{0.1} \mathrm{TiO}_{2.9} \mathrm{~F}_{0.1}, \mathrm{KBT}\right)$ is particularly interesting for its increased dielectric activity at room temperature, as shown in Figure $1 \mathrm{~b} .{ }^{41}$ Adding high- $\mathrm{k}$ dielectric nanoparticles to the PNIPAm hydrogels enables the hydrogels to exhibit a stronger response to an applied RF field.

The RF wave is remotely generated, which implies that the modulating signal will travel from the outside of the acoustic material medium. For a parallel plate antenna setup in which the material is between the plates, equation (1) above changes to

$$
p_{\mathrm{m}}\left(E_{\mathrm{a}}\right)=\omega \epsilon_{0} \epsilon_{\mathrm{r}}^{\prime} \cdot \tan \delta \cdot \frac{\left|E_{\mathrm{a}}\right|^{2}}{\epsilon_{\mathrm{r}}^{\prime 2}+\epsilon_{\mathrm{r}}^{\prime \prime 2}},
$$

where $E_{\mathrm{a}}$ is the applied RF electric field amplitude. Eventually, RF must be converted into heat to induce the change in the elastomechanical properties of the PNIPAm-based hydrogels used in this work. Using the equations above and thermodynamic laws for energy dissipation and heating, the heating rate of the material becomes

$$
\frac{\Delta T}{t}\left[\frac{{ }^{\circ} \mathrm{C}}{\mathrm{s}}\right]=\frac{5.56 e-11 \cdot f[\mathrm{~Hz}] \cdot \tan \delta \cdot\left|E_{\mathrm{a}}\left[\frac{\mathrm{V}}{\mathrm{m}}\right]\right|^{2}}{\rho_{\mathrm{m}}\left[\frac{\mathrm{kg}}{\mathrm{m}^{3}}\right] \cdot C_{\mathrm{p}}\left[\frac{\mathrm{J}}{\mathrm{kg}^{\circ} \mathrm{C}}\right] \cdot \epsilon_{\mathrm{r}}^{\prime}\left(1+(\tan \delta)^{2}\right)}
$$

From equation (3), it becomes apparent that the competing effects of dielectric responsiveness from the dielectric constant $\left(\varepsilon^{\prime}\right)$, loss tangent $(\tan \delta)$, density of the composite $\left(\rho_{\mathrm{m}}\right)$ and specific heat $\left(C_{\mathrm{p}}\right)$ must be appropriately considered to enable the sensitivity of a PNIPAm-based hydrogel to RF through additive dielectrics. Nanoparticle from $10 \% \mathrm{KF}-$ doped $\mathrm{BaTiO}_{3}$ (KBT) calcined at $800{ }^{\circ} \mathrm{C}$ was proposed as an ideal additive due to its dielectric properties near room temperature (Figure 1), as well as its stability in NIPA monomer solution. KBT nanoparticles were experimentally found to quickly precipitate out of water and the monomer solution used in bulk PNIPAm free-radical polymerization. The accelerated precipitation resulted in hydrogels with KBT concentrations that could not be accurately estimated based on the amount of KBT nanoparticles mixed into the monomer solution, as well as insufficient elastic properties in the KBT-hydrogel composite. To achieve the desired dispersion of the particles in the hydrogels, an additive was required to increase the viscosity of the monomer mixture without altering the tunable mechanical properties. PVA was selected as the additive as it lacks the appropriate vinyl groups to chemically bond to NIPA polymer chains and thus can be diluted out of the hydrogel. It 
was experimentally determined to sufficiently prevent the precipitation of KBT nanoparticles while maintaining tunable elastic properties inherent to PNIPAm. For this work, we refer to the materials with adequate RF sensitivity and elastic tenability as acoustic materials.

Infiltrating the acoustic material into a phononic crystal results in a device with material properties susceptible to change with RF-stimulation. This work details the study and development of KBT800 infused PNIPAm-based polymer materials for adequate RF sensitivity, the combining of the acoustic material with a phononic crystal to form the device and the coupling of control of transient ultrasound with applied RF light in the device. The RF susceptibility of the acoustic materials and evolution of the transmission band under RF stimulation are examined for an infiltrated structure to demonstrate the control of ultrasound using RF EM radiation.

\section{Hybrid phononic crystal}

The device is based on a phononic crystal of stainless steel cylindrical rod scatterers in a square lattice arrangement with a 50\% filling fraction for the unit cell. The base phononic crystal is effective in ambient water. The corresponding transmission spectrum from 200 to $800 \mathrm{kHz}$ at $0 \mathrm{~min}$ of RF exposure is shown in Figure 2a. We examined the effect of RF on the final device in both ambient air and water as water is susceptible to heating with applied RF.

The reduced frequency is defined as $\Omega=\omega /\left(c_{l} \cdot k\right)$, where $c_{l}$ is the velocity of sound in the propagating medium. The long-wavelength approximation for the base phononic crystal is $0<\Omega<0.56$ as indicated by its corresponding scaled band diagram in Figure $2 \mathrm{~b}$. This work focuses on the $450-650 \mathrm{kHz}$ range, which only contains elements outside the homogenization limit of the first transmission band (Figure 2b). The actual phononic crystal used for this work and an unscaled band diagram can be found in the Supplementary Figure S2.

Effects realized in the second band and above are considered metamaterial in nature as the crystal is no longer considered a homogeneous medium with respect to the impinging wave. Features that arise in transmission bands above the first are generally not functions of Bragg scattering, but rather of local resonances from topological properties of the scatterers and their contrast with the elastomechanical properties of the surrounding medium. ${ }^{42}$ Dimensions of the sonic crystal were selected to demonstrate the ability of RF to tune bulk structures as the total crystal dimension would be on the centimeter scale. Additionally, metamaterials effective near $0.5 \mathrm{MHz}$ in water may have applications for deeper imaging into soft tissues. In this device, spectral characteristics, defined here as the position, width and shape of the spectral bands, is a function of scatterer-ambient elastomechanical contrast as the size, shape and arrangement of the scatterers remains constant.

The dielectric properties of plain PNIPAm (Bulk Gel), PNIPAm formed using PVA without nanoparticles (PVA), and PNIPAm formed with PVA and dispersed KBT nanoparticles (KBT) were determined using RF dielectric spectroscopy. The density $\left(\rho_{\mathrm{m}}\right)$ and specific heat $\left(C_{\mathrm{p}}\right)$ of each composite were experimentally determined, incorporated into equation (3) and plotted in Figure 3. Plain bulk PNIPAm was compared with the hydrogels that incorporated PVA both with and without KBT nanoparticles. The full dielectric evaluation of the composites has been left for other works. Figure 3 shows that the addition of KBT to the hydrogel greatly increased both the dielectric constant $\left(\varepsilon^{\prime}\right)$ and tan $\delta$, compared with both the PVA and plain hydrogels. The process also increased the $C_{\mathrm{p}}$ and $\rho_{\mathrm{m}}$ compared with nanoparticle-free PVA hydrogel. Nanoparticle-free PVA demonstrates superior energy dissipation characteristics-an effect that was not expected in our initial hypothesis. The effects of the additives clearly show in Figure 3 that the addition of PVA (black) and KBT (KBT800, purple) to bulk PNIPAm (Bulk Gel, red) hydrogels enhances the RF sensitivity of the composite compared with standard PNIPAm. The inset within Figure 3 demonstrates the distribution of KBT nanoparticles in the PVA-PNIPAm hydrogel.

The non-evasive EM control of ultrasonic propagation through a phononic device is best demonstrated by the radio-frequency-induced modulation of the device's transmission band. Since hydrogel must remain hydrated, we investigated RF modulation both in ambient air, where drying can occur, and ambient water, where it is continually hydrated. The increased sound velocity in water as temperature rises has been noted to shift the transmission band but leave the band contour relatively unchanged due to its lack of frequency dispersive properties. ${ }^{22}$ Of particular interest is the band shape as changes illustrate modulation due to the elastic property changes of the hydrogel composite from exposure to RF. Three devices were made and evaluated based on the conclusions of the RF susceptibility of the acoustic materials discussed
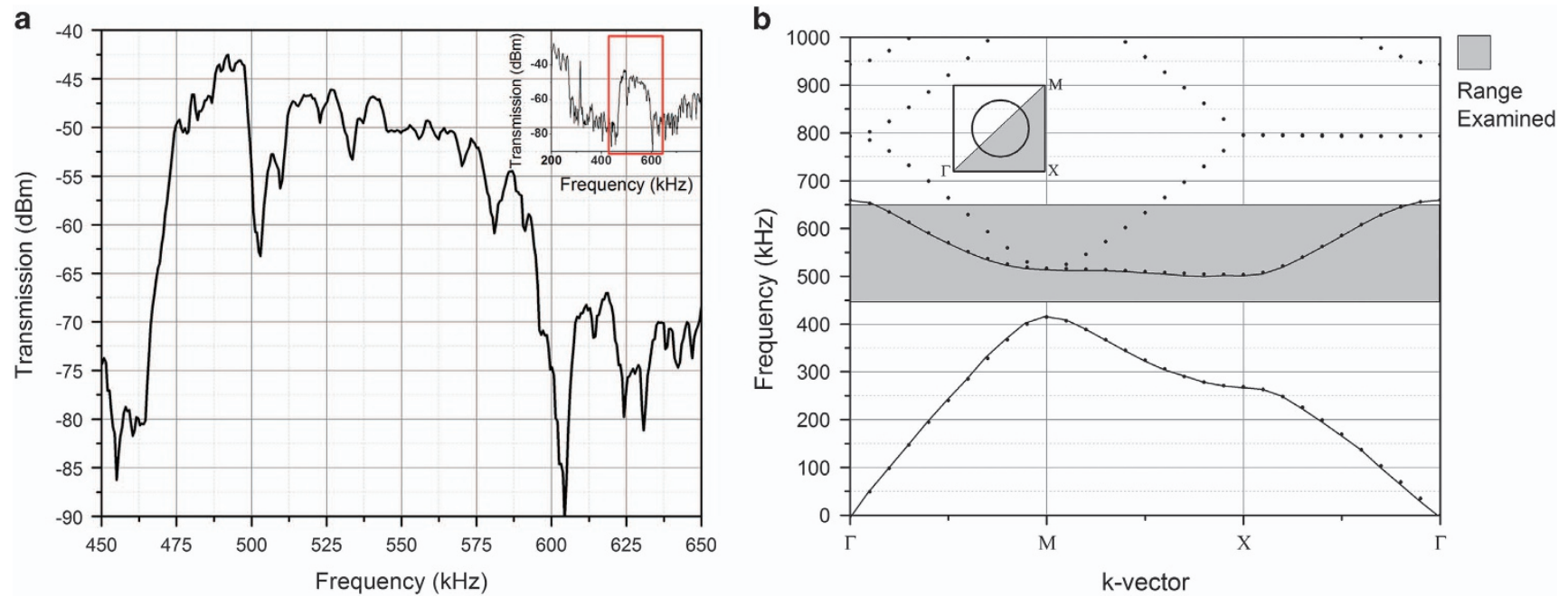

Figure 2 (a) The transmission spectrum for the base phononic crystal in water with an intraband pseudogap at $\sim 500 \mathrm{kHz}$. The inset is the spectrum 200-800 kHz and shows the transmission to be of the second band. (b) The full calculated band structure of the base phononic crystal in ambient water using Comsol 4.3 eigensolver with Matlab. The examined transmission band for the $\Gamma X$ is solidly above the homogenized regime for the crystal. 
a

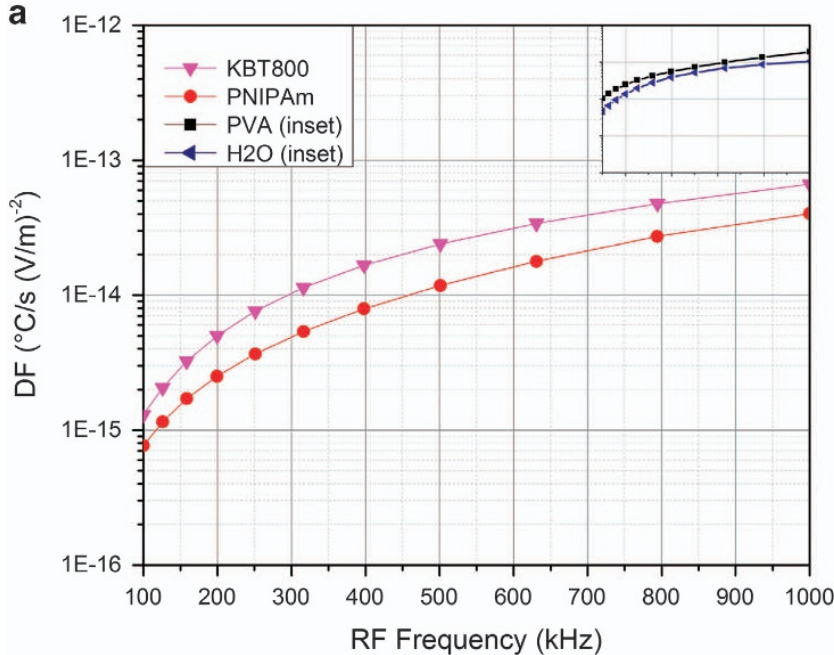

b

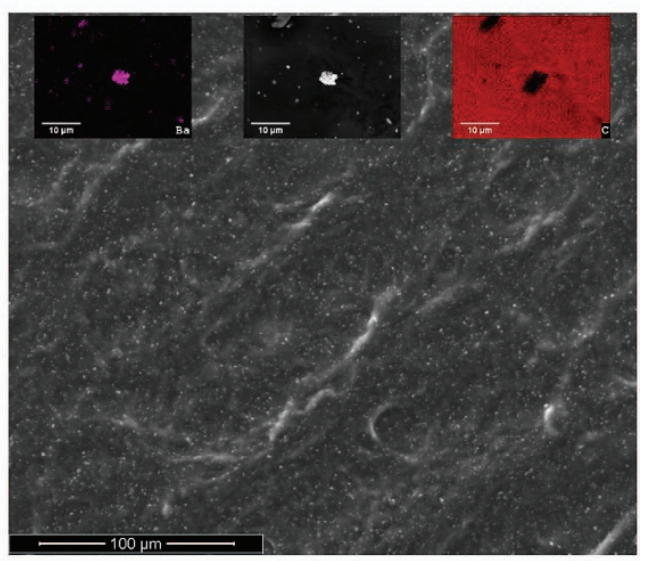

Figure 3 (a) Effective dissipation factor (DF) calculated using 3 from experimentally derived values. Corresponding DF values for PVA and $\mathrm{H}_{2} \mathrm{O}$ given in inset. KBT800 possess dispersed KBT in the hydrogels. (b) s.e.m. image of dispersed KBT nanoparticles in PVA-modified hydrogel at 15 kV and nanoparticle cluster (insets, scale bar $=10 \mu \mathrm{m}$ ).

in Figure 3: one without any hydrogel (SQSC), one with PVA-PNIPAm hydrogel (PVASC) and one with KBT nanoparticles dispersed PVAPNIPAm (KBTSC). As the bulk PNIPAm has relatively poor response compared with the other polymer composites, a standard bulk PNIPAm-based phononic device was not evaluated. Radio frequency waves were generated as outlined in the Supplementary Section. The contribution due to the water on the transmission of the sonic waves in the presence of an RF wave has been studied by investigating the phononic crystal in a water bath and has been presented in the Supplementary Section. As the PVA-PNIPAm hydrogel composite has a high RF susceptibility, a phononic device has also been tested for its response to RF actuation and is presented in the Supplementary Section.

KBT-hydrogel composite and water are both susceptible to heating with applied RF, with water ideally possessing an appreciable responsiveness (Figure 3 ). To distinguish the contribution of the surrounding water bath, which is susceptible to heating induced by RF waves, the KBT-hydrogel composite phononic device has also been studied in air.

The RF tuning of the device in ambient water has several interesting features. The effect of heating on the transmission spectrum of a phononic crystal composed of non-dispersive materials is primarily a shifting of the band with little change in the topology of intraband features. Figure $4 \mathrm{a}$ is the KBTSC ultrasonic transmission spectrum at the beginning $(0 \mathrm{~min})$, middle $(60 \mathrm{~min})$ and end $(120 \mathrm{~min})$ of the applied RF. The snapshots and select times show intraband pseudogaps not only shifting but also appearing and disappearing. The temporal evolution is scaled to $-10 \mathrm{~dB}$ from the maximum intensity detected (Figure $4 \mathrm{~b}$ ). It reveals broadening of the band from $115 \mathrm{kHz}$ to $124 \mathrm{kHz}$ and significant variance in the intensity of pseudoband features as RF is applied. The RF modulation factor, $\mathrm{M}(f, t)$, is defined as

$$
\mathbf{M}(f, t)=|P(f, t)-P(f, 0)| / P(f, 0),
$$

where $P(f, t)$ is the normalized measured signal in Watts at time $t$. The $\mathrm{RF}$ modulation of the low frequency band edge and large pseudogap that persists from $\sim 515$ to $\sim 545 \mathrm{kHz}$ is plotted in Figure 4c. High RF modulation factors can result from the transmission band shifting but are not necessarily indicative of changes in the topology of the transmission band. The Supplementary Information pertaining to water demonstrates this concept well. In the case of KBTSC, the combined inspection of Figure $4 \mathrm{~b}$ and $\mathrm{c}$ shows that the intraband features are not simply shifting due to increasing temperature in the ambient medium. The large modulation factors, reaching as high as 38 in this range, are the result of dispersive elastomechanical changes in the KBT hydrogel changing the topology of the transmission band and evidence of RF tuning of non-trivial metamaterial features.

Although RF in ambient water controls transient ultrasound as evidenced by the topological evolution of the second transmission band, the susceptibility of water to RF could mean that the control is trivial due to heating the ambient medium. Air does not maintain the same RF susceptibility, so evaluation in air provides additional information on RF control of sound in the device. Hydrogel is $>90$ wt. \% water, and thus can dry out when exposed to air for extended periods of time. Figure $5 \mathrm{a}$ contains the comparison of transmission in air with and without RF applied over a 10 min time span. The applied RF is clearly the source of modification of the transmission band as minimal changes occur without it.

For the ambient air study, RF was applied for $50 \mathrm{~min}$ then turned off and the transmission measured for the same amount of time. Unlike the case of ambient water, there are no clear pseudogaps in the transmission band (Figure 5b). However, the ultrasonic transmission spectrum is strongly modified as evidenced by an expansion of nearly $8.5 \%$ (Figure $5 \mathrm{~b}$ ). The phase reversibility of the hydrogel component is demonstrated in Figure $5 \mathrm{c}$ and $\mathrm{d}$ as the transmission features retreat towards their initial values when RF application is removed. The significance of the changes can be more closely examined at the high frequency band edge where modulation factors nearing 650 are reached (Figure $5 \mathrm{~d}$ ). The measurements began immediately after the removal of the device from ambient water so that it was fully hydrated. The device was not rehydrated for the duration of the experiments, which is the likely cause of the variation between the spectrum at the onset of RF and after the RF was removed and the hydrogel was allowed to return to its initial state.

\section{DISCUSSION}

Metamaterial devices maintain synthetic properties achieved outside the long wavelength approximation of periodic or semi-periodic structures. The properties achievable in the above devices are functions of the k-vector of the impinging sound wave, the contrast in elastic properties between scatterers and the ambient medium, and the 
a

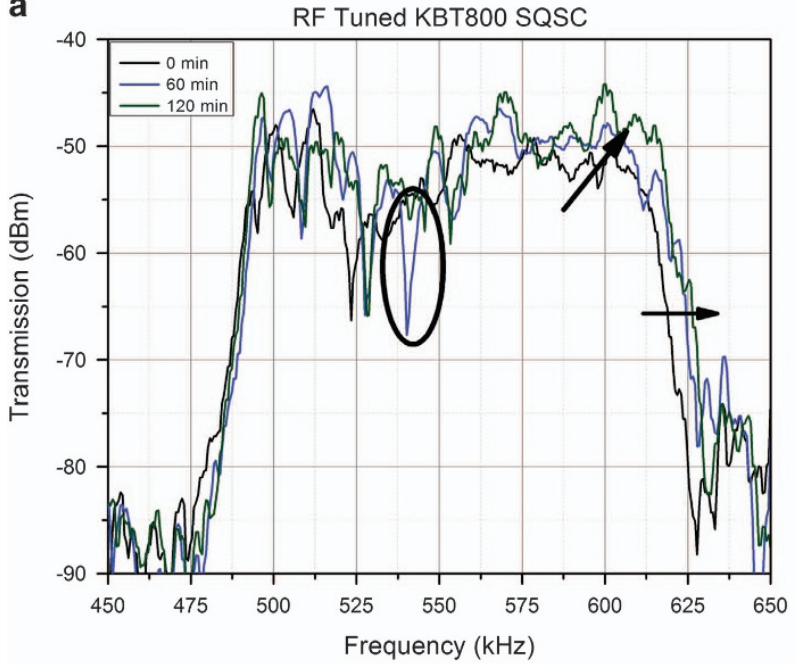

b

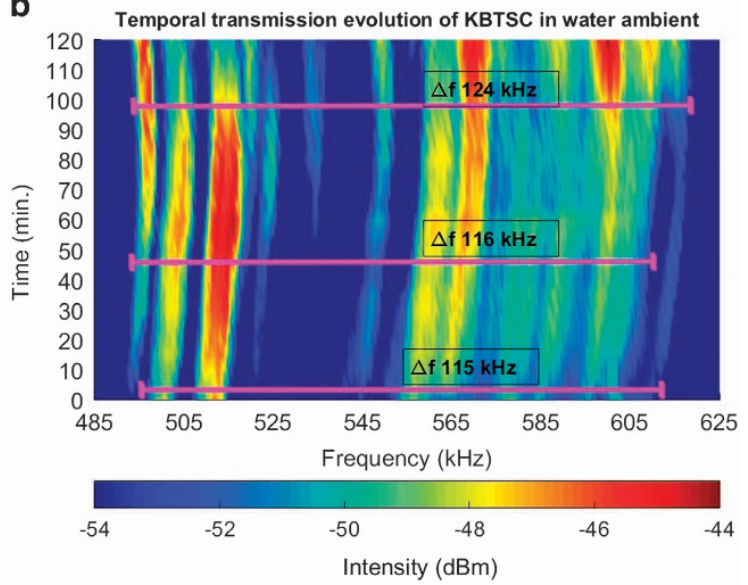

C KBTSC

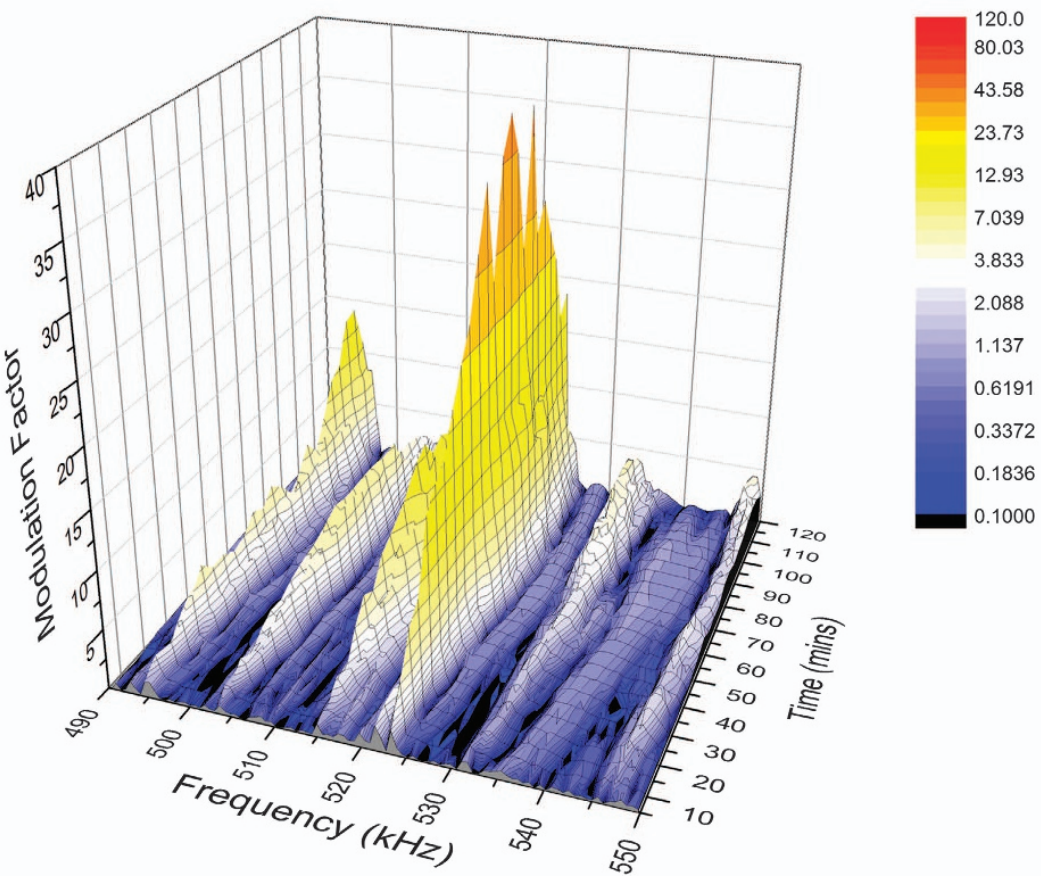

Figure 4 The transmission of KBTSC in ambient water. (a) The spectrum of KBTSC at 0 (black), 60 (blue) and 120 (green) min of RF application. The expansion (arrows) and pseudoband features (circle) are due to dispersive changes in elastomechanical properties of KBT hydrogel. (b) Temporal intensity evolution shows band expansion by $7.8 \%$ with no shifting of the lower band edge after 5 min of applied RF. (c) Intraband topological changes evidenced by modulation factor.

elastomorphic or topological characteristics of the scatterer and lattice of the component phononic crystal. ${ }^{4,43}$ The spectral profile, or shape of the transmission band, indicates the transient behavior of ultrasound, and in this case is modulated by applying RF. The effect of radio waves on water, which exists as an ambient medium, was considered as it is susceptible to RF stimuli, and the analysis was performed both in ambient water and air.

SQSC is the phononic crystal component of the device without RF actuated acoustic material interstitially filling the crystal and is presented in the Supplementary Materials. The Supplementary Information includes an examination and commentary on the temperature-induced frequency shift. The spectrum of SQSC blue shifts due to the RF heating the ambient water. Similar shifts have also been demonstrated in arrangements where heat was applied to ambient water through other means. ${ }^{22}$ For the temperature increase induced by the RF field, the relative contrast in sound velocity between the stainless steel scatterers and ambient water is maintained over the full spectral range due to water being a low-dispersion medium. Hybridization and other resonance features that arise in a transmission band are not principally functions of lattice spacing, but of contrast in elastomechanic properties and local elastomorphic features. Inspection of the leading and trailing band edges, and of intraband features in the water-filled phononic, reveals shifts of $\sim 8 \mathrm{kHz}$, supporting the contention that the contour shape is maintained (Supplementary Figure S3). Though shifting due to RF in this scenario technically performs as an RF susceptible metamaterial, only a shift in the effective frequency range occurs. A dispersive change in the elastomechanic contrast leads to dynamic change in the dispersion relation 
a

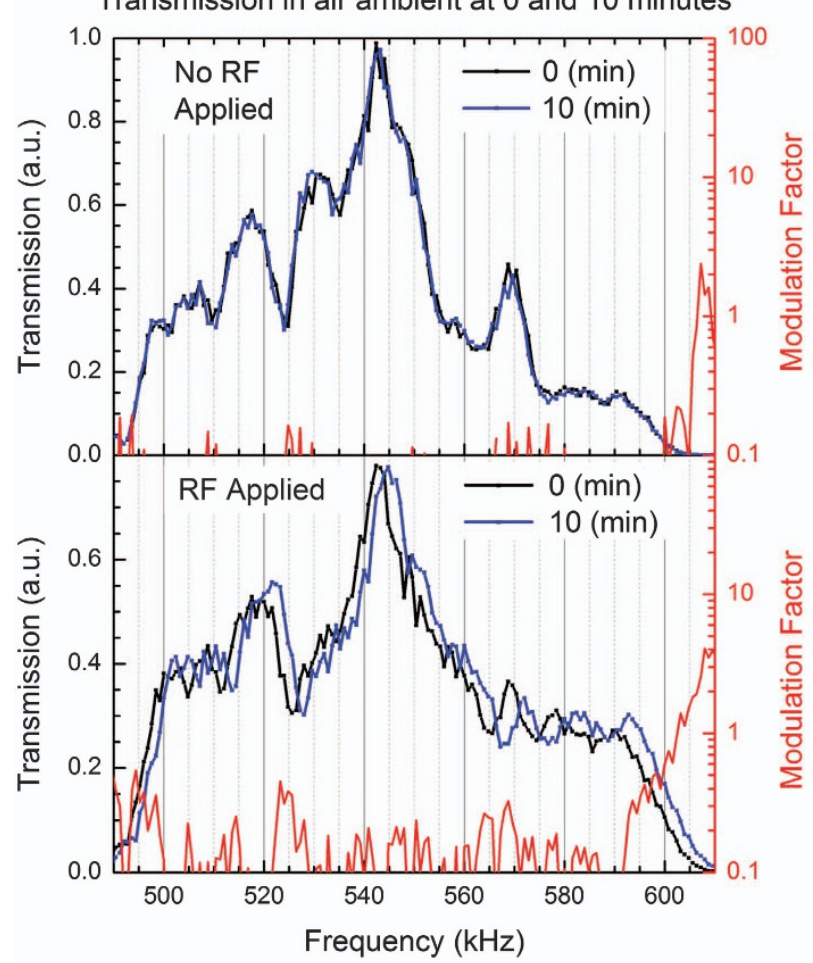

b

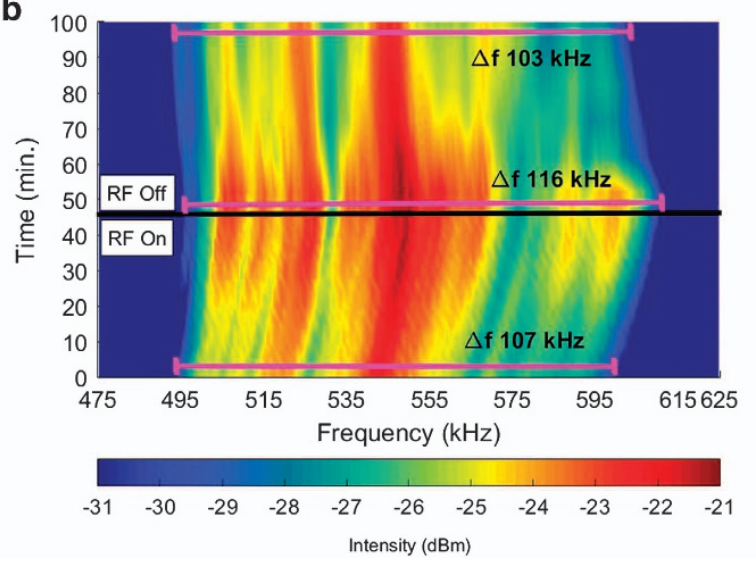

c

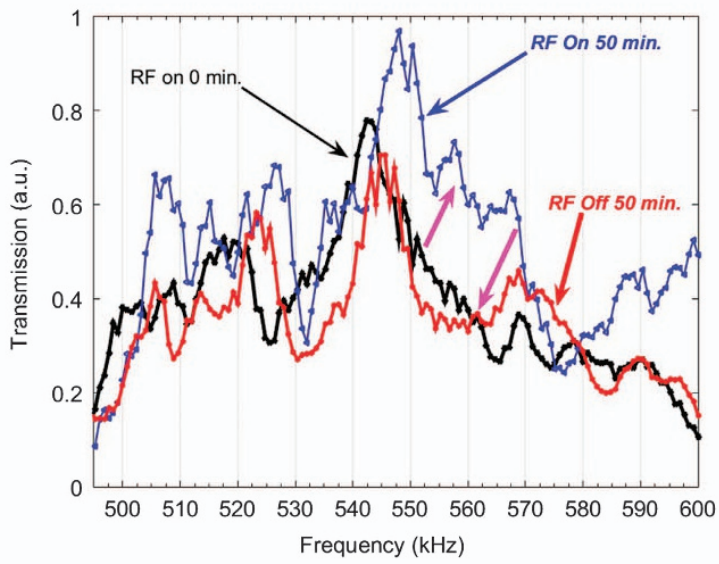

d

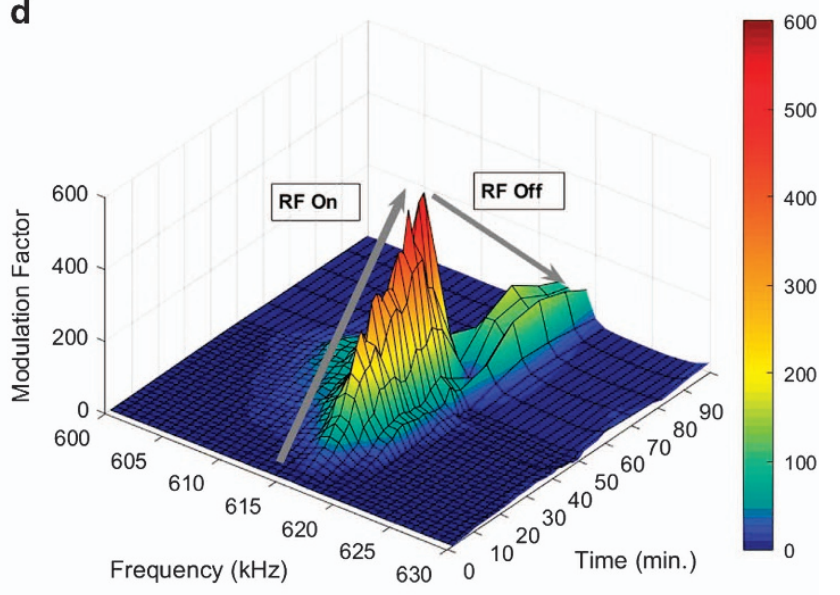

Figure $5 \mathrm{KBTSC}$ transmission in ambient air. (a) Evaporation of water from KBTSC shows negligible effects at 10 min, while the applied RF induces shifts and begins band expansion. (b) Temporal intensity evolution does not reveal any strong pseudobands, but it does show band expansion by $8.4 \%$ (c) The reversibility of the process is illustrated in the return of the transmission towards the initial values after RF has been removed. (d) Modulation factor at high-frequency band edge where the increase in intensity is due to additional available eigensolutions due to local resonances. 44,45

in the phononic device, as indicated by the transmission spectrum. This effect can potentially result in a device with metamaterial properties that are only activated or deactivated with applied RF.

For KBTSC, the pseudobands and intraband features that arise from hybridization and other non-Bragg resonances are heavily modified due to the application of RF. The dispersive temperature-dependent elastic properties of the polymer results in ephemeral features that include increases and decreases in spectral width and intensity as well as shifting. The full characterization of the frequency-dependent elastomechanical properties of KBT-dispersed PVA PNIPAm or other PNIPAm-based materials in the ultrasonic frequency regime is necessary to model the device band diagram accurately. Accurate modeling would enable the design of more functional narrow-band metamaterials such as RF tunable ultrasonic cloaks, filters or negative index lenses.
In ambient air, the strongest modulation of KBTSC occurs at the high-frequency band edge, essentially resulting in a tunable switch that turns on with RF and off without it. Band edges are usually of great importance in the design of metamaterials as they are highly nonlinear and can lead to anomalous effects. ${ }^{44}$ Though RF also heats the ambient medium with KBTSC in water, the potential for a functional narrow-band metamaterial is greater due to the modulation that occurs in the $\sim 520$ to $\sim 545 \mathrm{kHz}$ frequency range, in addition to the expansion at the high-frequency band edge.

In summary, this work demonstrates the control of ultrasound waves in a phononic crystal-based metamaterial device via RF stimulation. The ability of RF to penetrate deeply into bulk structures made it the ideal mechanism for the regulation of sound through a structure. High-k nanoparticles and PVA were dispersed into PNIPAm-based hydrogels to 
make a material that possessed elastomechanical properties, such as speed of sound and elastic modulus, susceptible to RF stimulation. Control was accomplished by incorporating the material into a phononic crystal and modulating the elastomechanical contrast with RF. Metamaterial properties were significantly altered as evidenced by the modulation factor of the second spectral transmission bands in KBTSC in ambient air and water reaching upwards of 550 and expansion by nearly $8 \%$. Materials with adjustable elastomechanic properties that also either intrinsically or artificially respond to EM stimulus provide a clear mechanism for more exotic devices. The response time is a function of the hydrogel dielectric, heat transport and elastomechanic temperature dependence in addition to the applied frequency and ambient medium. Though this work did not seek to optimize response times, faster responses can be achieved through both material optimization and higher RF application frequencies. Resulting devices fundamentally couple EM control to exotic acoustic properties.

\section{CONFLICT OF INTEREST}

The authors declare no conflict of interest.

\section{ACKNOWLEDGEMENTS}

The authors gratefully acknowledges Prof. Y. Akishige, Shimane University, Japan for help with the synthesis of $\mathrm{KF}-\mathrm{BaTiO}_{3}$ nanoparticles. The support from UNT China Venture funds is also acknowledged.

1 Cho, G., Jung, M., Yang, H., Lee, B. \& Song, J. H. Photonic crystals with tunable optical stop band through monodispersed silicapolypyrrole core-shell spheres. Mater. Lett. 61, 1086-1090 (2007).

2 Aydin, K., Bulu, I. \& Ozbay, E. Subwavelength resolution with a negative-index metamaterial superlens. Appl. Phys. Lett. 90, 254102 (2007).

3 Cai, W., Chettiar, U. K., Kildishev, A. V. \& Shalaev, V. M. Optical cloaking with metamaterials. Nat. Photonics 1, 224-227 (2007).

4 Kushwaha, M. S., Halevi, P. \& Martinez, G. Theory of acoustic band structure of periodic elastic composites. Phys. Rev.B 49, 2313-2322 (1994).

5 Sainidou, R., Stefanou, N., Psarobas, I. E. \& Modinos, A. A layer-multiple-scattering method for phononic crystals and heterostructures of such. Comput. Phys. Commun. 166, 197-240 (2005).

6 Krokhin, A. A., Arriaga, J. \& Gumen, L. Speed of sound in periodic elastic composites. Phys. Rev. Lett. 91, 264302 (2003).

7 Bucay, J., Roussel, E., Vasseur, J. O., Deymier, P. A., Hladky-Hennion, A. C., Pennec, Y., Muralidharan, K., Djafari-Rouhani, B. \& Dubus, B. Positive, negative, zero refraction, and beam splitting in a solid/air phononic crystal: theoretical and experimental study. Phys. Rev. B 79, 214305 (2009).

8 Yang, S., Page, J. H., Liu, Z., Cowan, M. L., Chan, C. T. \& Sheng, P. Focusing of sound in a 3D phononic crystal. Phys. Rev. Lett. 93, 024301 (2004).

9 Goffaux, C., Maseri, F., Vasseur, J. O., Djafari-Rouhani, B. \& Lambin, P. Measurements and calculations of the sound attenuation by a phononic band gap structure suitable for an insulating partition application. Appl. Phys. Lett. 83, 281-283 (2003).

10 Centini, M., Sibilia, C., Scalora, M., Aguano, G. D., Mertolotti, M., Bloemer, M. J., Bowden, C. M. \& Nefedov, I. Dispersive properties of finite, one-dimensional photonic band gap structures: applications to nonlinear quadratic interactions. Phys. Rev. E 60, 4891-4898 (1999).

11 Yeh, J. Y. Cavity characteristic analysis of two-dimensional phononic crystal. J. Eng. Technol. Educ. 8, 393-399 (2011).

12 Kosevich, Y. A., Goffaux, C. \& Sanchez-Dehesa, J. Fano-like resonance phenomena by flexural shell modes in sound transmission through two-dimensional periodic arrays of thin-walled hollow cylinders. Phys. Rev. B 74, 012301 (2006).

13 Kaina, N., Lemoult, F., Fink, M. \& Lerosey, G. Negative refractive index and acoustic superlens from multiple scattering in single negative metamaterials. Nature 525, 77-81 (2015).

14 Yeh, J. Y. Control analysis of the tunable phononic crystal with electrorheological material. Physica B 400, 137-144 (2007).

15 Wang, Y., Song, W., sun, E., Zhang, R. \& Cao, W. Tunable passband in one-dimensional phononic crystal containing a piezoelectric $0.62 \mathrm{~Pb}(\mathrm{Mg} 1 / 3 \mathrm{Nb} 2 / 3) \mathrm{Or}-0.38 \mathrm{PbTiO3}$ single crystal defect layer. Physica E 60, 37-41 (2014).

16 Zhou, X. \& Chen, C. Tuning the locally resonant phononic band structures of two-dimensional periodic electroactive composites. Physica B 431, 23-31 (2013).

$17 \mathrm{Xu}, \mathrm{Z}$., Wu, F. \& Guo, Z. Shear-wave band gaps tuned in two-dimensional phononic crystals with magnetorheological material. Solid State Commun. 154, 43-45 (2013).
18 Malinovsky, V. \& Donskoy, D. Electro-magnetically controlled acoustic metamaterials with adaptive properties. J. Acoust. Soc. Am. 132, 2866-2872 (2012).

19 Robillard, J. F., Matar, O. B., Vasseur, J. O., Deymier, P. A., Stippinger, M., Hladky-Hennion, A. C., Pennec, Y. \& Djafari-Rouhani, B. Tunable magnetoelastic phononic crystals. Appl. Phys. Lett. 95, 124104 (2009).

20 Wang, J., Xu, X., Liu, X. \& Xu, G. A tunable acoustic filter made by periodical structured materials. Appl. Phys. Lett. 94, 181908 (2009).

21 Goffaux, C. \& Vigneron, J. P. Theoretical study of a tunable phononic band gap system. Phys. Rev. B 64, 075118 (2001).

22 Walker, E., Reyes-Contreras, D., Rojas, M. M., Krokhin, A., Wang, Z. \& Neogi, A. Tunable ultrasonic phononic crystal controlled by infrared radiation. Appl. Phys. Lett. 105,143503 (2014).

23 Kushwaha, M. S., Djafar-Rouhani, B., Dobrzynski, L. \& Vasseur, J. O. Sonic stop-bands for cubic arrays of rigid inclusions in air. Eur. Phys. J. B 3, 155-161 (1998).

24 Cheng, W., Wang, J., Jonas, U., Fytas, G. \& Stefanou, N. Observation and tuning of hypersonic bandgaps in colloidal crystals. Nat. Mat. 5, 830-836 (2006).

25 Eichenfield, M., Chan, J., Camacho, R., Vahala, K. \& Painter, A. Optomechanical crystals. Nature 462, 78-82 (2009).

26 Alonso-Redondo, E., Schmitt, M., Urbach, Z., Hui, C. M., Sainidou, R., Rembert, P., Matyjaszewski, K., Bockstaller, M. R. \& Fytas, G. A new class of tunable hypersonic phononic crystals based on polymer-tethered colloids. Nature Commun. 6, 8309 (2015).

27 Walker, E., Reyes, D., Krokhin, A. \& Neogi, A. Anomalous temperature dependence of speed of sound of bulk poly(N-Isopropylacrylamide) hydrogels near the phase transition. Ultrasonics 54, 1337-1340 (2014).

28 Budhlall, B. M., Marquez, M. \& Velev, O. D. Microwave, photo- and thermally responsive PNIPAm-gold nanoparticle microgels. Langmuir 24, 11959-11966 (2008).

29 DeRosa, M., DeRosa, R., Noni, L. \& Hendrick, E. Phase separation of poly(N-isopropylacrylamide) solutions and gels using a near infrared fiber laser. J. Appl. Polymer Sci. 105, 2083-2090 (2007).

30 Garcia, A., Marquez, M., Cai, T., Rosario, R., Hu, Z., Gust, D., Hayes, M., Vail, S. A. \& Park, C. D. Photo-, thermally, and pH-responsive microgels. Langmuir 23, 224-229 (2007).

31 Juodkazis, S., Mukai, N., Wakaki, R., Yamaguchi, A., Matsuo, S. \& Misawa, H. Reversible phase transitions in polymer gels induced by radiation forces. Nature 408, 178-181 (2000).

32 Ishikawa, M., Misawa, H., Kitamura, N., Fujisawa, R. \& Masuhara, H. Infrared laserinduced photo-thermal phase transition of an aqueous poly( $\mathrm{N}$-isopropylacrylamide) solution in the micrometer dimension. Bull. Chem. Soc. Jpn 69, 59-66 (1996).

33 Tomatsu, I., Peng, K. \& Kros, A. Photoresponsive hydrogels for biomedical applications. Adv. Drug Deliv. Rev. 63, 1257-1266 (2011).

34 Ramaswamy, H. \& Tang, J. Microwave and radio frequency heating. Food Sci. Technol. 14, 423 (2008).

35 Zhao, Y., Flugstad, B., Kolbe, E., Park, J. \& Wells, J. Using capacitive (radio frequency) dielectric heating in food processing and preservation-a review. J. Food Process Eng. 23, 25-55 (2000).

36 Zhou, J., Wei, J., Ngai, T., Wang, L., Zhu, D. \& Shen, J. Correlation between dielectric/ electric properties and cross-linking/charge density distributions of thermally sensitive spherical PNIPAM microgels. Macromolecules 56, 6158-6167 (2012).

$37 \mathrm{Wu}, \mathrm{C}$. A comparison between the 'coil-to-globule' transition of linear chains and the 'volume phase transition' of spherical microgels. Polymer 39, 4609-4619 (1998).

38 Cho, E. C., Lee, J. \& Cho, K. Role of bound water and hydrophobic interaction in phase transition of poly(N-isopropylacrylamide) aqueous solution. Macromolecules $\mathbf{3 6}$, 9929-9934 (2003).

39 Akishige, Y. Ferroelectric and piezoelectric properties of single crystals of $\mathrm{Ba}_{(1-x)} \mathrm{K}_{(\mathrm{x})}$ $\mathrm{TiO}_{(3-\mathrm{x})} \mathrm{F}_{(\mathrm{x})}$ with $\mathrm{x}=0.1$. J. Phys. Soc. Jpn 75, 073704 (2006).

40 Tsukada, S., Hiraki, Y., Akishige, Y. \& Kojima, S. Enhanced polarization fluctuation in KF-substituted BaTiO3 single crystals. Phys. Rev. B 80, 012102 (2009).

41 Akishige, Y., Hiraki, Y., Tsukada, S., Xu, J., Morio, S., Ohba, T., Walker, E. \& Neogi, A. Dielectric and piezoelectric properties of $10 \%$ KF-doped BaTiO3 ceramics. Jpn J. Appl. Phys. 49, 081501 (2010).

42 Page, J. H., Lee, E. J. \& Croënne, C. Anomalous ultrasonic transport in phononic crystals with overlapping Bragg and hybridization gaps. J. Acoust. Soc. Am. 134, 4026 (2013).

43 Nedelec, J. C. Acoustic and Electromagnetic Equations (Springer, 2001).

44 Szmulowicz, F. New Kronig-Penney equation emphasizing the band edge conditions. Eur. J. Phys. 29, 507-515 (2008).

45 Sainidou, R. \& Stefanou, N. Formation of absolute frequency gaps in three-dimensional solid phononic crystals. Phys. Rev. B 66, 212301 (2002).

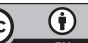

This work is licensed under a Creative Commons Attribution 4.0 International License. The images or other third party material in this article are included in the article's Creative Commons license, unless indicated otherwise in the credit line; if the material is not included under the Creative Commons license, users will need to obtain permission from the license holder to reproduce the material. To view a copy of this license, visit http:// creativecommons.org/licenses/by/4.0/

(C) The Author(s) 2017

Supplementary Information accompanies the paper on the NPG Asia Materials website (http://www.nature.com/am) 